\title{
Efficiency of the Gaver-Stehfest Method Transient Response of a Spiral Fin
}

\author{
Bilal Sungur ${ }^{* *}\left(\mathbb{D}\right.$, Ibrahim Keles ${ }^{2}$ (i) \\ 1,2Department of Mechanical Engineering, Samsun University, 55420, Samsun, Turkey
}

\begin{abstract}
In this study, a practical combined approach was applied by the Gaver-Stehfest method to examine the impermanent reaction of a spiral fin whose tip is isolated with the base tip exposed to changes in fluid temperature. The heat transmission of the fin under the stable temperature effect of the base is examined. The environment temperature is saved stationary, and no heat source or sink is available. Results are improved for minor- and major -time worth when the base is exposed to the unit step variation form in temperature. The changes in heat flux and temperature at the base formed depending on the parameters that make up the spiral fin are graphically shown. The available numerical results show that it is fully consistent when compared with the literature.
\end{abstract}

Keywords: Laplace transform, Transient response, Spiral fin, Heat transfer, Gaver-Stehfest method

\section{INTRODUCTION}

Many engineers and researchers have done a lot of work to increase the response problem in the fins and heat dissipation from a hot surface using fins in industrial applications. One of the methods used to raise the amount of heat transfer with the developing technology is the fins used to expand the heat transfer surfaces. Spiral fins are widely used in industrial applications and are used to ensure optimum temperature distribution in turbines, generators, power plants, and electronic devices [1].

Chu et al. [1-3] analyzed the impermanent responses of both external flat and annular fins as well as unidimensional circular fin and compound flat fins using the Fourier sequences and overturn method. Using the natural convection correlations available for plates, the efficiency of circular fins of distinct profiles exposed to local heat transmission constant as a function of local temperature was investigated by Mokheimer et al. [4]. The impact of conduction heat transmission in different shapes of annular fins [5], current conduction effect in multidimensional sizes [6], fins exposed to temperature-addicted heat flux [7], and dispersed transportation effect along the curly plaque consisting of circular slices [8] have been studied by the researchers. Also, problems related to the optimization of fins and spines have been investigated $[9,10]$. Yu and Chen [11] recommended the Taylor transform and limited difference approach to examine the non-linear temporary heat trans- mission problematic of the rectangular profile circular fin, taking into account the step temperature change occurring in the radiation wing tip and infinite base by convection. By choosing the heat transmission constant temperature as the power function type and using the power values representing changed heat transmission machines for example independent convection, completely industrialized boiling and radiation, the longitudinal and annular fins and spines have been presented by Laor and Kalman [12]. Zubair et al. [13] analyzed a one-dimensional fin with a variant shape that can be abridged to a rectangular shape. Bouaziz et al. [14] examined the temperature-dependent efficacy of longitudinal fins by thermo-physical features. The symbolic mathematical equation for the tip temperature and fin efficacy of circular blades, depending on the thermo-geometric blade parameters and radius ratio, was obtained by Campo and Stuffle [15]. Wang et al. [16] analytically presented the temporary reply of a spiral fin whose bottom was subjected to variant heat flow.

Suppose the solution of mathematical, physics, chemistry and engineering problems defined by differential equations is resolved in the Laplace space. In that case, it may be hard or even unbearable to gain an analytical translation into the time domain. Numerical methods for numerical overturn of the inverse Laplace transform to generate resolutions in the time area have been used in science and engineering since the 1930s. The Gaver-Stehfest method is one of these
* Corresponding author

Email: bilal.sungur@samsun.edu.tr
European Mechanical Science (2021), 5(4): 194-200

doi: https://doi.org/10.26701/ems.899389

Received: July 3, 2020

Accepted: September 8, 2020 
numerical methods that is used efficiently and successfully. Since the introduction of the method, the Gaver-Stehfest procedure has been successfully implemented in many fields such as geophysics [17], probability [18,19], actuarial mathematics [20], and scientific finance [21], chemical [22], and economy [23].

In this study, the solutions of the temporary reply of the end insulated spiral fin are analyzed for unit step temperature change subject to change by the base in fluid temperature with easy-to-calculate Laplace transformations for short time intervals. After the general equations that make up the problem are obtained in the Laplace space, the results of the temperature change obtained by transporting them to the time space by the Gaver-Stehfest method are presented in graphs. It can be said that the results given in Ref.[16] and the results obtained here match exactly.

\section{TRANSIENT RESPONSE MATHEMATICAL MODEL OF A SPIRAL FIN}

Fig. 1 demonstrated a spiral fin's schematic diagram with livery thickness $\mathrm{z}=2 \delta$, internal radius ri, outside radius ro, field $\mathrm{P}$, and thermal conductivity $k$. The end of the fin, $r=r_{o}$ is supposed well isolated. Besides, a one-dimensional analysis assumption is made. At first, the fin and the surrounding fluid temperature $\left(T_{\infty}\right.$ are in thermal equilibrium. The bottom temperature is instantly increased to $T_{f}$ and related to heat flux $q_{0}^{*}$, since then, the spiral fin dissolute heat to the perimeter via convection mechanism. The properties $h, h_{\rho}$ and $c, \rho, k$ of the fin materials are all accepted as constant. In additional boundary condition, $r=r$, by ignoring the thermal resistor and capacity of the physical in internal wall pipe, the convective boundary condition is assumed.

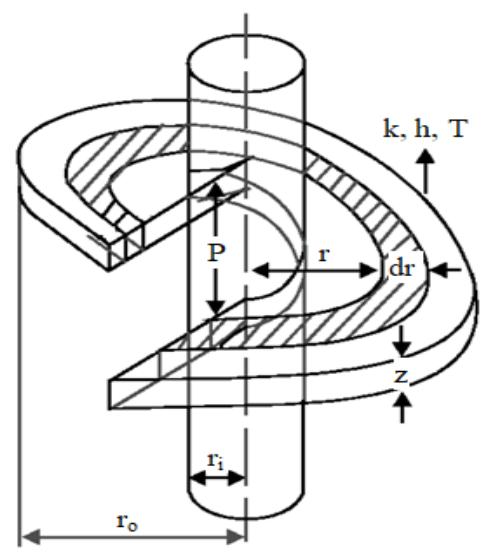

Figure 1. Schematic view of the spiral fin [16]

Using the conservation of energy equation, the differential equation of the spiral fin temperature can be written as[16]:

$$
\frac{\partial}{\partial \mathrm{r}}\left\{\sqrt{\left(\frac{P^{2}}{2 \pi}\right)+r^{2}} \frac{\partial \mathrm{T}}{\partial \mathrm{r}}\right\}-\frac{h}{k \delta} \sqrt{\left(\frac{P^{2}}{2 \pi}\right)+r^{2}}\left[T-T_{\infty}\right]=\frac{1}{\alpha} \sqrt{\left(\frac{P^{2}}{2 \pi}\right)+r^{2}} \frac{\partial \mathrm{T}}{\partial \mathrm{r}}, t>0, r_{i}<0<r_{o}
$$

where, $\alpha=k / \rho c$ is the thermal diffusivity and $B_{i}=h_{f} r_{i} / k_{f}$ is the Biot number.

After the explanation of non-dimensional parameters,

$$
\xi=\frac{r}{r_{i}}, \phi=\frac{T-T_{\infty}}{T_{f}-T_{\infty}}, \alpha=\frac{\alpha t}{r_{i}^{2}}, P_{i}=\frac{P}{2 \pi r_{i}}, R=\frac{r_{o}}{r_{i}}, N^{2}=\frac{h r_{i}^{2}}{k \delta}
$$

The non-dimensional governing equation becomes as follows:

$$
\frac{\partial}{\partial \xi}\left\{\sqrt{P_{i}^{2}+\xi^{2}} \frac{\partial \phi}{\partial \xi}\right\}-N^{2} \sqrt{P_{i}^{2}+\xi^{2}} \phi=\sqrt{P_{i}^{2}+\xi^{2}} \frac{\partial \phi}{\partial \tau}, \tau>0, \quad 1<\xi<R
$$

The environmental temperature is assumed that at a fixed temperature, and it was also assumed that there was no heat source or heat sinks. The non-dimensional initial and boundaries can be explained as follows:

$$
\begin{aligned}
& \phi(\xi, 0)=0,1<\xi<R \\
& -\frac{\partial \phi}{\partial \xi}+B_{i} \phi=B_{i}, \quad \xi=1, \quad \tau>0 \\
& -\frac{\partial \phi}{\partial \xi}(R, t)=0, \quad \tau>0
\end{aligned}
$$

Eq.2 is subjected to Laplace transform, and by applying the initial condition given in Eq.3, the final state of the equation is as follows:

$$
-\frac{\partial \phi}{\partial \xi}\left\{\sqrt{P_{i}^{2}+\xi^{2}} \frac{\partial \bar{\phi}}{\partial \xi}\right\}-\left\{\sqrt{P_{i}^{2}+\xi^{2}}\left[N^{2}+s\right]\right\} \bar{\phi}=0
$$

In this equation, s symbolized the transformed variant. Integrals in the exponential constant can be neglected, and then the Keller and Keller's solution of Eq. 6 becomes as follows:

$$
\bar{\phi}(\xi, s)=D_{2}\left\{-\left[N^{2}+s\right]\left[P_{i}^{2}+\xi^{2}\right]\right\}^{-1 / 4} \cdot \cosh \left[\sqrt{N^{2}+s}(R-\xi)\right]
$$

In this equation, $D_{2}$ is a fixed to be identified by the boundary condition (4). The boundary condition (4) can be expressed as follows by using Laplace transformation:

$$
-\frac{\partial \bar{\phi}}{\partial \xi}+B_{i} \bar{\phi}=\frac{B_{i}}{s}, \quad \xi=1
$$

The constant $D_{2}$ can be expressed as follows by substituting Eq.7 into Eq.8,:

$$
D_{2}=\frac{2 B_{i}\left[N\left(N N^{2} s\right)\right]^{1 / 4}\left[P_{i}^{2}\right]^{3 / 2}}{\left\{2\left[P_{i}^{2}+1\right]^{3 / 4} \cdot \sqrt{N^{2}+s} \cdot s \cdot \sinh \left[\sqrt{N^{2}+s}(R-1)\right]+s\left[P_{i}^{2}+1\right]^{1 / 4} \cdot \cosh \left[\sqrt{N^{2}+s}(R-1)\right]\left[2 B_{i}\left(P_{i}^{2}+1\right)+1\right]\right\}}
$$

Thus, Eq.7 becomes

$$
\bar{\phi}(\xi, s)=\frac{2\left[P_{i}^{2}+1\right]^{s / 4}}{s\left[P_{i}^{2}+\xi^{2}\right]^{14}} \frac{\cosh \left[\sqrt{N^{2}+s}(R-\xi)\right]}{\left\{2\left[P_{i}^{2}+1\right] \cdot \sqrt{N^{2}+s} \cdot s \cdot \sinh \left[\sqrt{N^{2}+s}(R-1)\right]+\left[2 B\left(P^{2}+1\right)+1\right] \cdot \cosh \left[\sqrt{N^{2}+s}(R-1)\right]\right\}}
$$

Defining the dimensionless heat flux at the fin base as:

$$
q_{0}^{*}=q_{b} r_{1} / 4 k\left[\delta \pi r_{1}\right] T_{f}-T_{\infty}
$$

After using the dimensionless constraints, the heat flux can be written as:

$$
q_{0}^{*}=-\sqrt{P_{i}^{2}+1} \frac{\partial \phi}{\partial \xi}(1, \tau), \quad \tau>0
$$

\section{THE GAVER-STEHFEST INVERSE LAPLACE METHOD}

Researchers have successfully applied the Gaver-Stehfest method(GSM) to many different fields and different prob- 
lems and based on a simple algorism for numerical overturn of the Laplace transform [24-26].

With this method, the Laplace space is transformed into the time domain as follows [27];

$$
f(t)=\frac{\ln 2}{t} \sum_{k=1}^{M} V_{k} F\left(k \frac{\ln 2}{t}\right)
$$

where $V_{k}$ is expressed as follows;

$$
V_{k}=(-1)^{k+\frac{M}{2}} \sum_{j=\left(\frac{k+1}{2}\right)}^{\min \left(k \frac{M}{2}\right)} \frac{j^{\frac{M}{2}}(2 j) !}{\left(\frac{M}{2}-j\right) ! j !(j-1) !(k-j) !(2 j-k) !}
$$

Here the parameter $M$ is expressed as the number of Stehfest. The precision of the calculation depends on the M value, so this value must be an even integer because the inversion is based upon the sum of $M$ weighted values. In theory, choosing a large value for $\mathrm{M}$ would suggest a more correct resolution, but if $\mathrm{M}$ is too big, results may deteriorate because of rounding mistakes. Therefore, it is important to choose an appropriate $\mathrm{M}$ value to reach the best solution [28]. Most researchers suggest a changed worth of the M constraint to get the best solution. Cheng and Sidauruk [29] suggested that the $M$ value should be between 6 and 20. In this study, the M value was chosen as 16 .

\section{RESULTS}

Dimensionless distribution of temperature of the spiral fin found from Eq. 7 is given in Fig. 2-5 for distinct worthies of $B_{i}, \tau, R$ and $P_{i}$. It can be seen from these figures, in all cases, the temperature distribution, $\phi$, rises with the increasing time. Additionally, while the effect of the increase in time is more effective at the beginning, it gradually decreases afterward. This means the throughput heat flux in base of spiral fin will be reduced and the internal temperature of spiral fin will be increased with the time elapsed. Also, the increase in pitch values $P_{i}$ increased the temperature distribution at the similar values of $\tau, R, B_{i}$, and $N$ excepting for the high values of $N$ and low values of $\tau$.

When we examine the Fig. 2 and Fig. 3, at the larger value of $B_{i}$, the maximum temperature distribution obtained at the similar values of $N, P_{i}, \tau$ and $R$. Increasing the $B_{i}$ number means that the heat transmission from the fin base will increase, and in this case, it is one of the important parameters affecting the temperature distribution. In order to compare the effect of the $\mathrm{N}$ value, it will be useful to analyze Fig. 3 and Fig. 4. These figures demonstrated that the higher values of $N$, means the lesser temperature distribution at the similar values of $\tau, P_{i}, R$ and $B_{i}$. The effect of the $R$ values is shown from Fig. 3 and Fig. 5. It can be said that similar trends were obtained as $N$ value, while the $R$ rises, the temperature distribution declines at the similar value of $\tau, B_{i}, P_{i}$ and $N$.

As shown in Fig. 2-5, in all cases, it has been determined that the numerical calculation results were in well arrangement with the analytical consequences obtained from the literature [16].

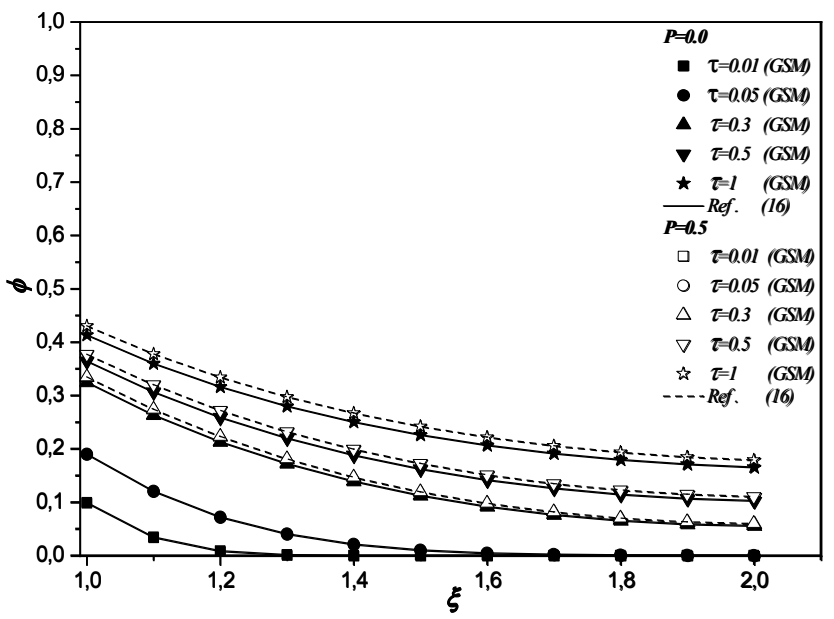

Figure 2. Distributions of temperature for varying $\tau$ with $R=2, N=1$ and $B_{i}=1$

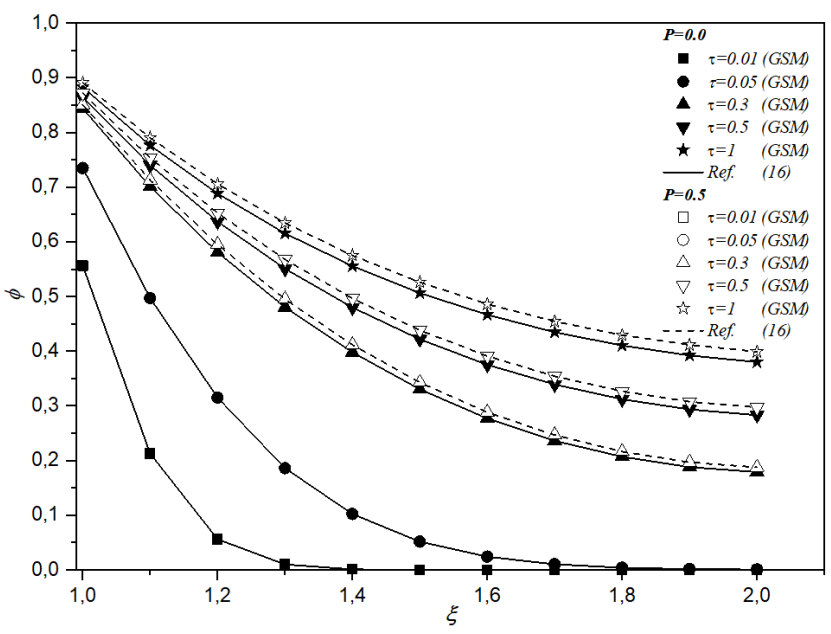

Figure 3. Distributions of temperature for varying $\tau$ with $R=2, N=1$ and $B_{i}=10$

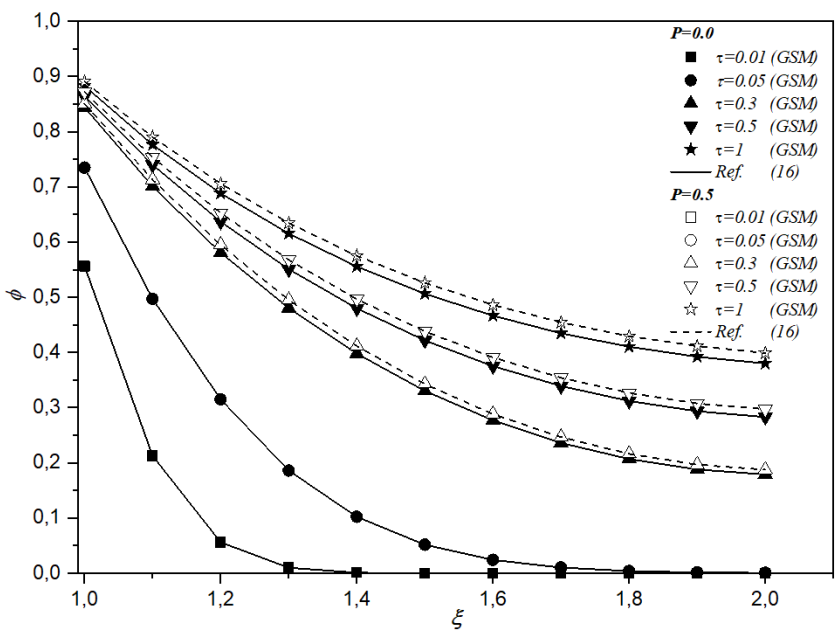

Figure 4. Distributions of temperature for varying $\tau$ with $R=2, N=5$ and $B_{i}=10$

The heat flux at the fin base, $q_{0}^{*}$, with distinct constraints of т, $B_{i}, P_{i}$ and $N$ are given in Fig. 6 and Fig.7. As shown from these figures, increasing the $N$ number increased the heat flux values in all cases. Also, increasing the $B_{i}$ number increased the heat flux values in all cases. After a certain time 
$(\tau \approx 1)$, the effect of time becomes almost negligible. Additionally, increasing the $P_{i}$, increased the heat flux values for all Bi and $\mathrm{N}$ numbers.

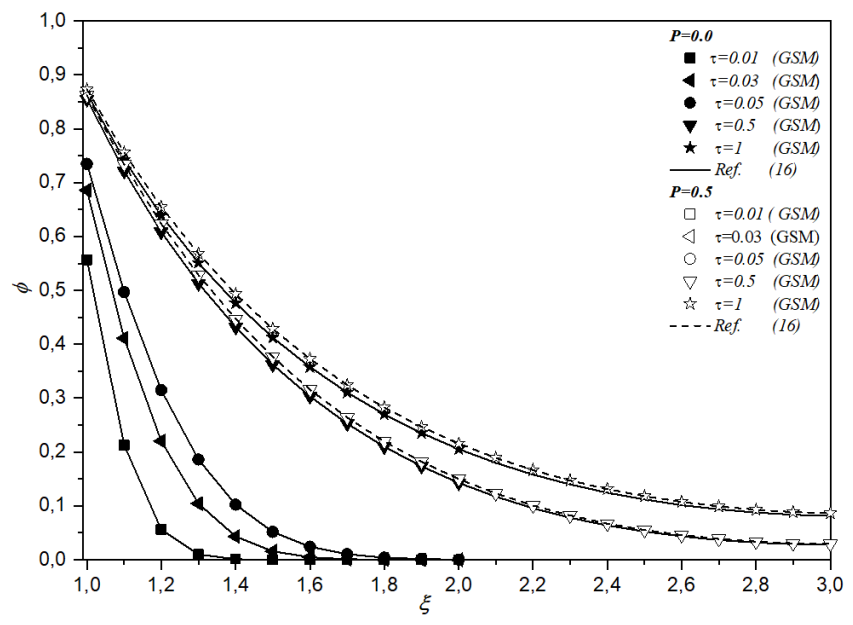

Figure 5. Distributions of temperature for varying $\tau$ with $R=3, N=1$ and $B_{i}=10$

The variation of temperature distribution with time is given in Fig. 8 and Fig. 9 for different $B_{i}$, and $\mathrm{N}$ numbers. As can be seen, there are very similar trends in both graphs and the effect of $P_{i}$ to the temperature distribution is very small in

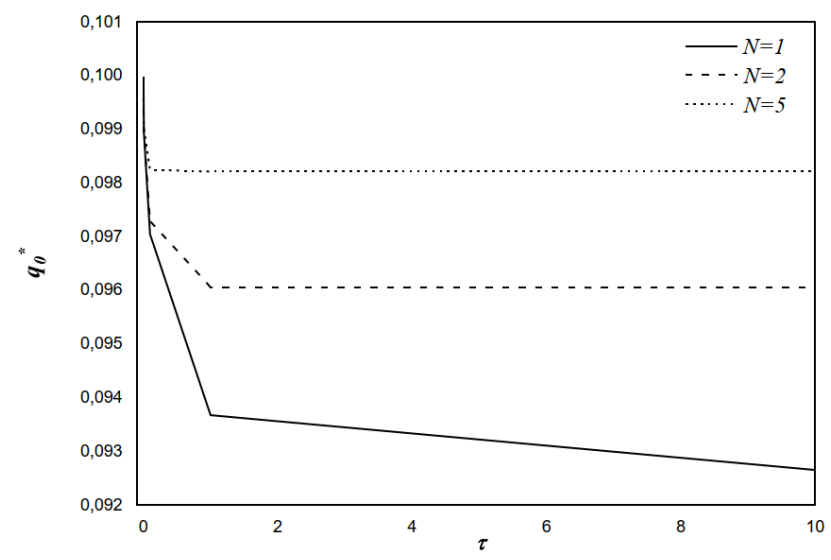

$(B i=0.1)$

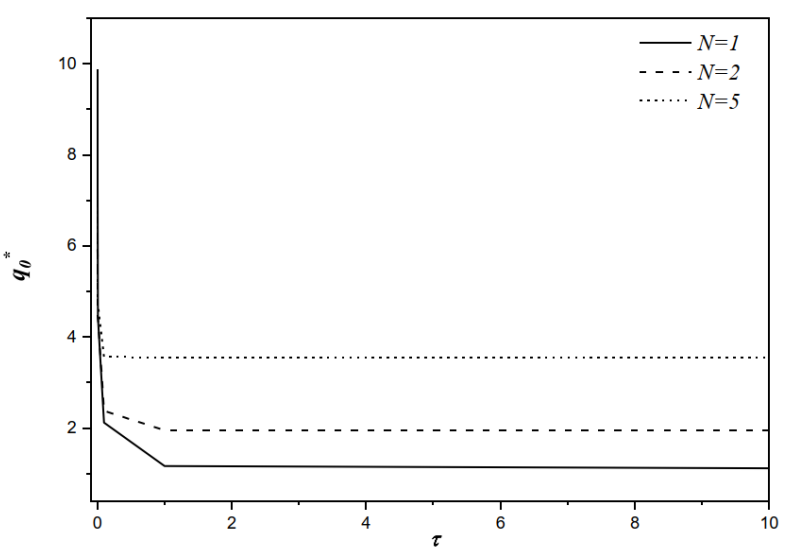

$(B i=10)$ these conditions. Increasing the $B_{i}$ number increased the heat transfer and for this reason, it is seen that the temperature progresses faster with at higher $B_{i}$ numbers. Also, the higher $\mathrm{N}$ number means the higher heat transfer rate and temperature distribution. However, with the increase in the number of $\mathrm{Bi}$, the effect of the $\mathrm{N}$ number gradually decreased, and the curves got closer to each other.

\section{CONCLUSIONS}

Numerical model of the transient response of the end insulated spiral fin for temperature and heat flux distributions are obtained and solved by GSM. When the efficiency and adequacy of the current method are compared with the analytical results, it is seen that they overlap [16] one to one. The solution technique and procedure are simple, efficient, and well structured and provide low-cost accuracy. When the numerical results in this study are evaluated, the results are briefly summarized below:

- The technique applied is more effective than the conservative techniques.

- The results show that the temperature change is affected depending on the $N, P_{\imath} R$ variables.

- The larger the $N$ value, the greater the heat conversion creates the spiral blade and the greater the tem-

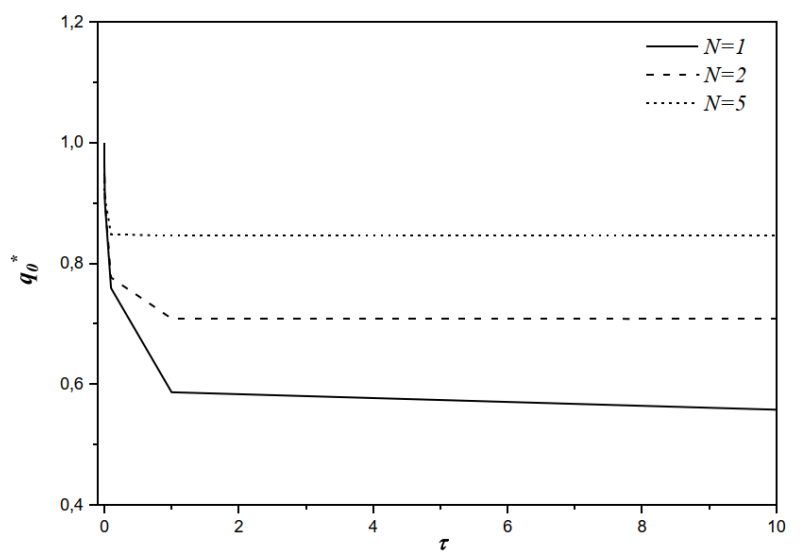

$(B i=1)$

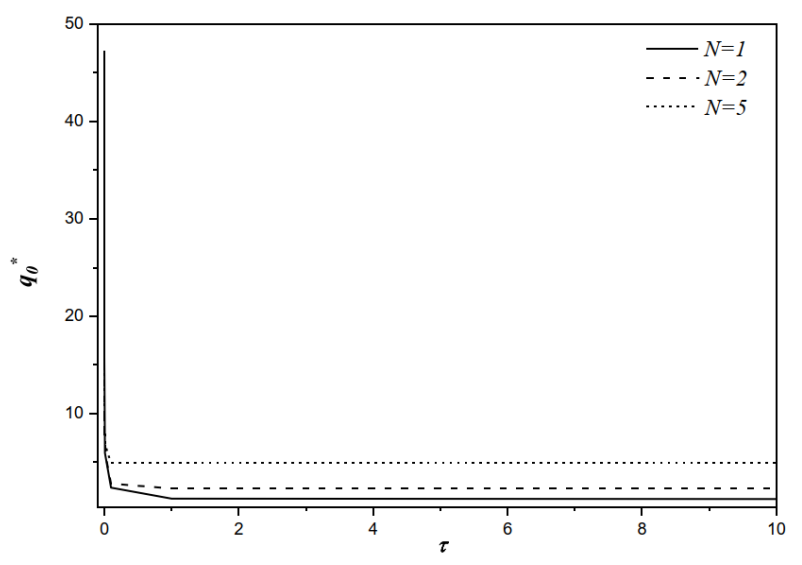

$(B i=50)$

Figure 6. Distributions of heat flux for varying $N$ with $R=2$, and $P_{i}=0$ 

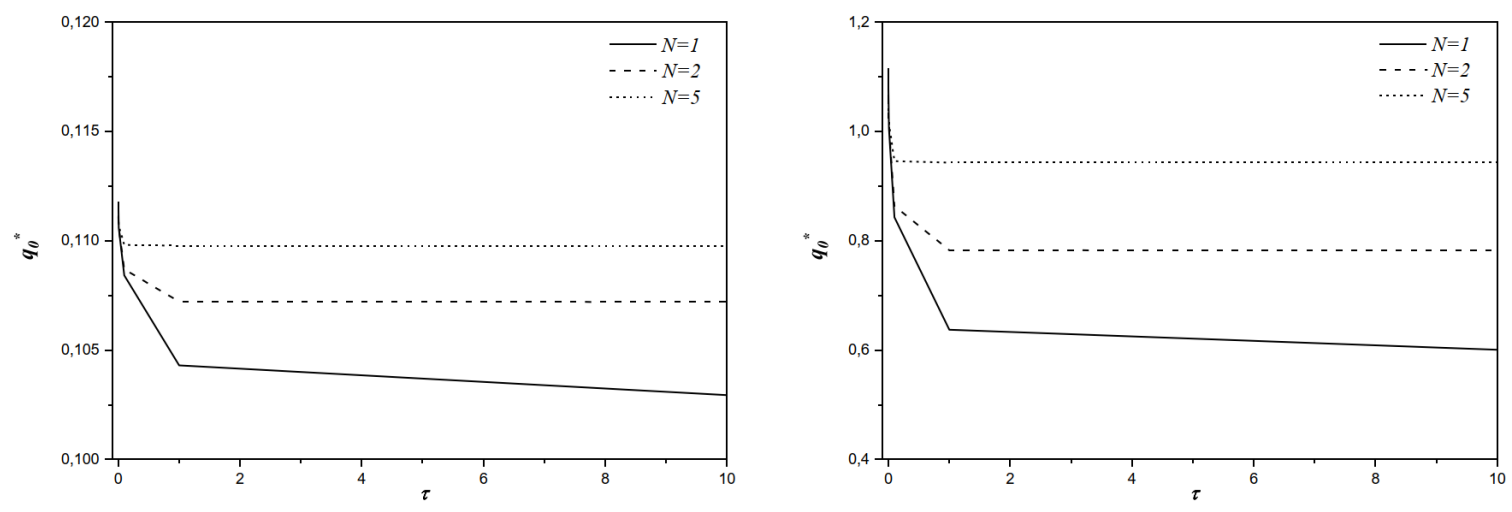

$(B i=0.1)$

$(B i=1)$

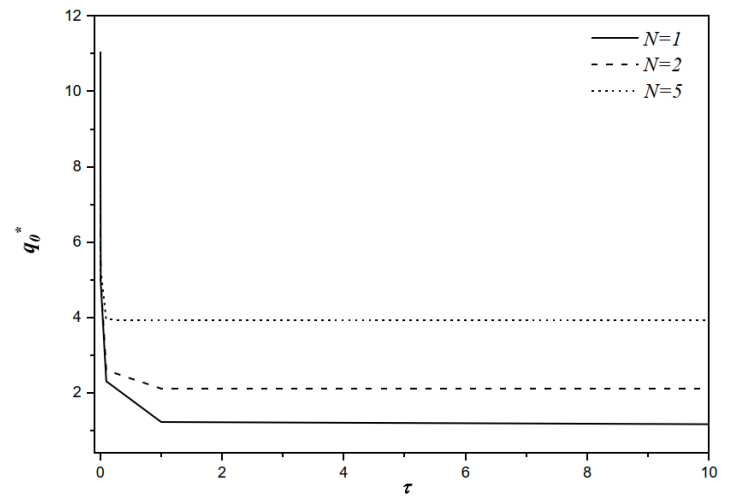

$(B i=10)$

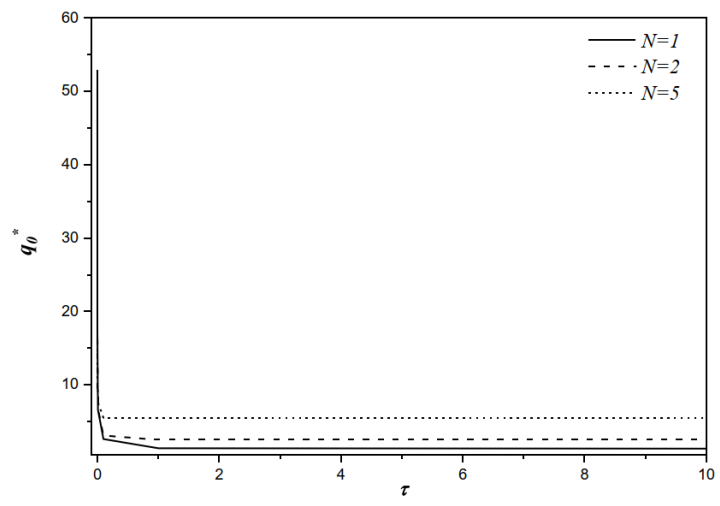

$(B i=50)$

Figure 7. Distributions of heat flux for varying $N$ with $R=2$, and $P_{i}=0.5$

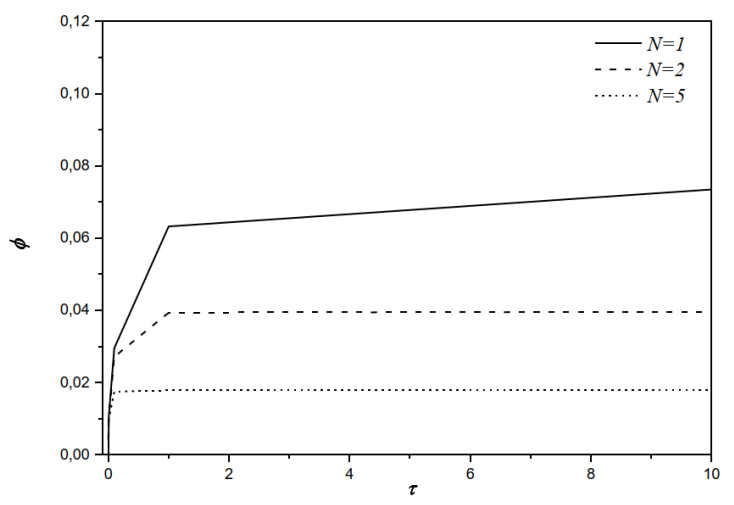

$(B i=0.1)$

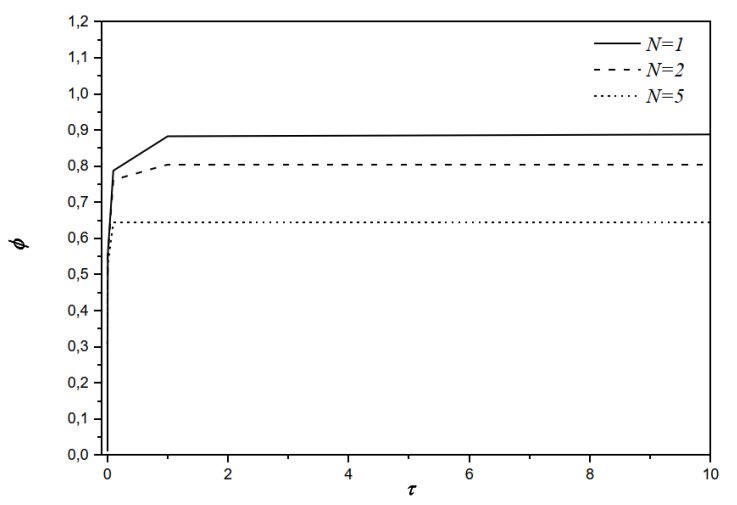

$(B i=10)$

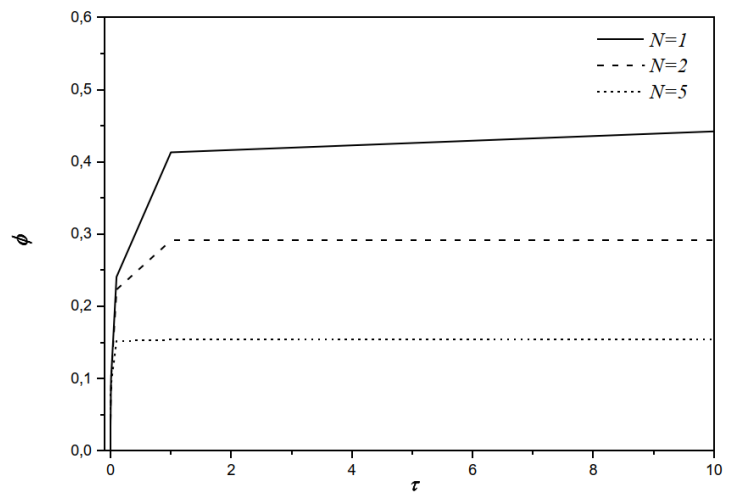

$(B i=1)$

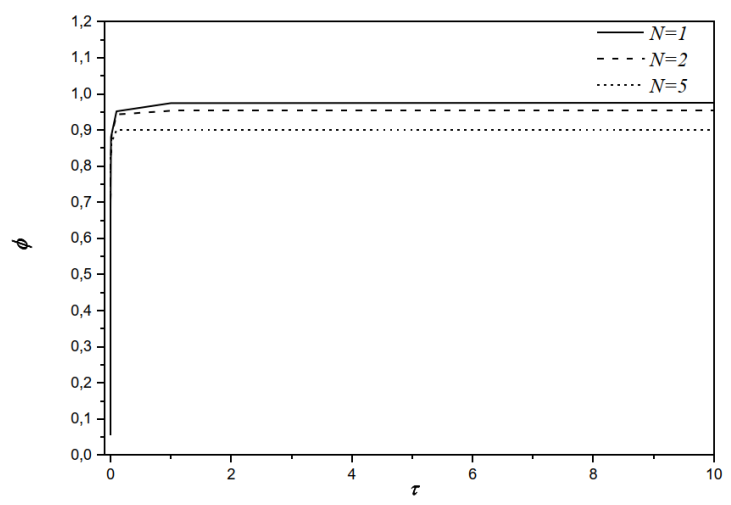

$(B i=50)$

Figure 8. Distributions of temperature for varying $N$ with $R=2$, and $P_{i}=0$ 


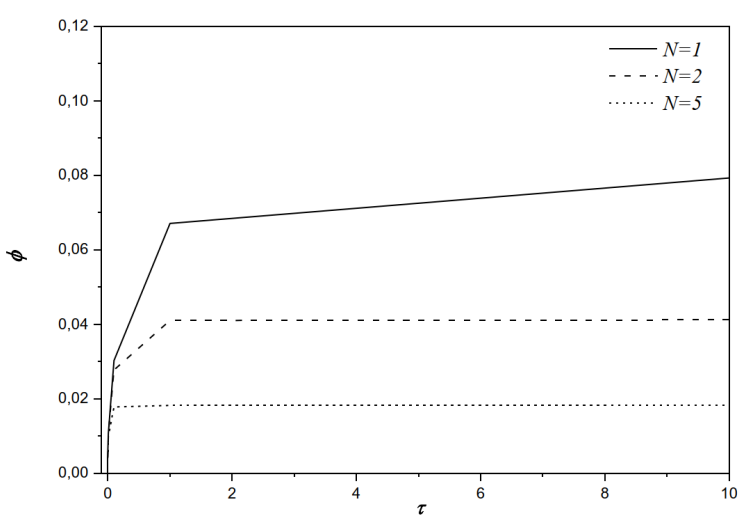

$(B i=0.1)$

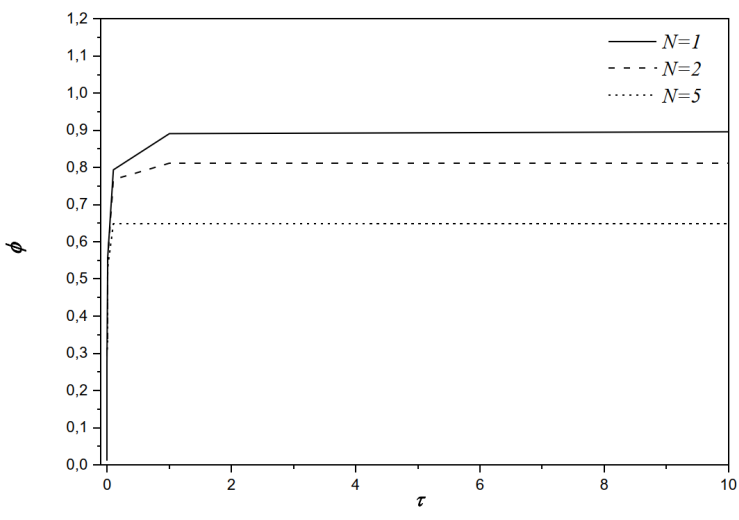

$(B i=10)$

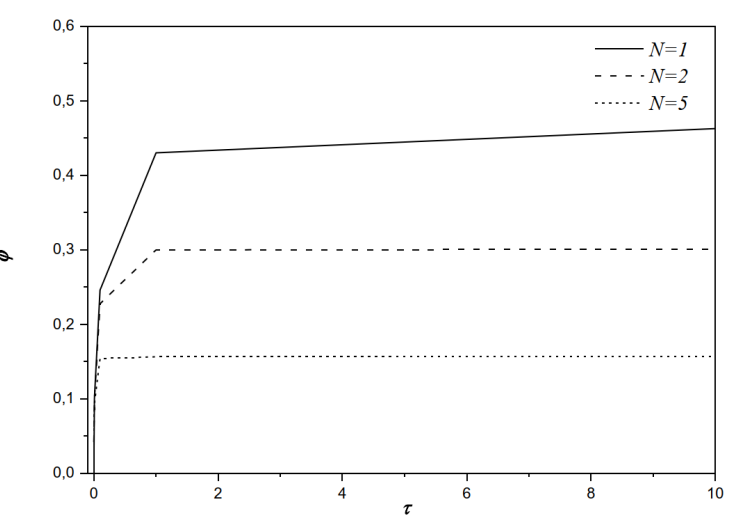

$(B i=1)$

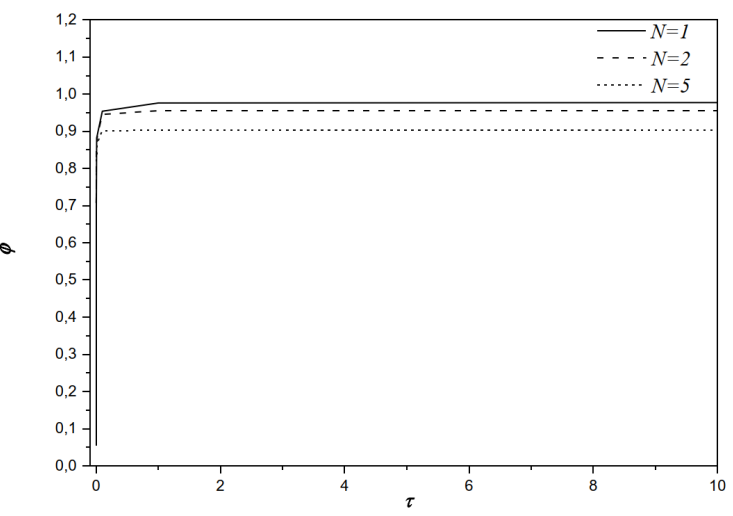

$(B i=50)$

Figure 9. Distributions of temperature for varying $N$ with $R=2$, and $P_{i}=0.5$

perature distribution.

- For the heat flux distribution $q_{0}^{*}$ in the spiral fin base, the variable $N$ and $P_{i}$ are the important variables, and the variable $P_{i}$ affects $q_{0}^{*}$ much more as the $N$ variable increases.

- The results obtained in this study can be used as a basis in the application of spiral fins to the industry.

\section{REFERENCES}

[1] Chu, H.-S., Chen, C.-K., Weng, C.-I., (1982). Applications of Fourier series technique to transient heat transfer problem. Chemical Engineering Communications. 16(1-6): 215-25. https://doi. org/10.1080/00986448208911098

[2] Chu, H.S., Chen, C.K., Weng, C.I., (1983). Transient response of circular pins. Journal of Heat Transfer. 105(1): 205-8. doi: 10.1115/1.3245547.

[3] Chu, H. Sen., Weng, C.I., Chen, C.K., (1983). Transient Response of Composite Straight Fin. Advances in the Astronautical Sciences. 50(pt 1): 539-49. https://doi.org/10.1115/1.3245579

[4] Mokheimer, E.M.A., (2002). Performance of annular fins with different profiles subject to variable heat transfer coefficient. International Journal of Heat and Mass Transfer. 45(17): 3631-42. https://doi. org/10.1016/S0017-9310(02)00078-9

[5] Cheng, C.-Y., (1994). Transient response of annular fins of various shapes subjected to constant base heat fluxes. Journal of Physics D: Applied Physics. 27(11): 2302. https://doi.org/10.1088/00223727/27/11/009

[6] Lee, Y.-M., Haji-Sheikh, A., Fletcher, L.S., Peterson, G.P., (1994). Effective thermal conductivity in multidimensional bodies. Journal of
Heat Transfer. 116(1):17-27. https://doi.org/10.1115/1.2910853

[7] Liaw, S.P., Yeh, R.H., (1994). Fins with temperature dependent surface heat flux-1. Single heat transfer mode. International Journal of Heat and Mass Transfer. 37(10): 1509-15. https://doi.org/10.1016/00179310(94)90152-X

[8] Wang, C.Y., (1994). Diffusive transport across a wavy plate composed of annular sectors.

[9] Yeh, R.-H., (1994). Optimum spines with temperature dependent thermal parameters. International Journal of Heat and Mass Transfer. 37(13): 1877-84. https://doi.org/10.1016/0017-9310(94)90328-X

[10] Yeh, R.-H., (1994). Optimization of longitudinal fins with temperature-dependent thermal parameters. Heat Transfer Engineering. 15(3): 25-34. https://doi.org/10.1080/01457639408939828

[11] Chen Cha'O-Kuang, L.-T.Y., (1999). Application of the Taylor transformation to the transient temperature response of an annular fin. Heat Transfer Engineering. 20(1): 78-87. https://doi. org/10.1080/014576399271736

[12] Laor, K., Kalman, H., (1996). Performance and optimum dimensions of different cooling fins with a temperature-dependent heat transfer coefficient. International Journal of Heat and Mass Transfer. 39(9): 1993-2003. https://doi.org/10.1016/0017-9310(95)00296-0

[13] Zubair, S.M., Al-Garni, A.Z., Nizami, J.S., (1996). The optimal dimensions of circular fins with variable profile and temperature-dependent thermal conductivity. International Journal of Heat and Mass Transfer. 39(16): 3431-9. https://doi.org/10.1016/00179310(96)00011-7

[14] Bouaziz, M.N., Rechak, S., Hanini, S., Bal, Y., Bal, K., (2001). Étude des transferts de chaleur non linéaires dans les ailettes longitudinales. International Journal of Thermal Sciences. 40(9): 843-57. https:// 
doi.org/10.1016/S1290-0729(01)01271-6

[15] Campo, A., Stuffle, R.E., (1997). Symbolic mathematics for the calculation of thermal efficiencies and tip temperatures in annular fins of uniform thickness. International Journal of Heat and Mass Transfer. 40(2): 490-2. https://doi.org/10.1016/0017-9310(96)00093-2

[16] Wang, J.S., Luo, W.J., Hsu, S.P., (2008). Transient Response of a Spiral Fin with its Base Subjected to the Variation of Heat Flux. Journal of Applied Sciences. 8(10): 1798-811. https://doi.org/10.3923/ jas.2008.1798.1811

[17] Knight, J.H., Raiche, A.P., (1982). Transient electromagnetic calculations using the Gaver-Stehfest inverse Laplace transform method. Geophysics. 47(1): 47-50. https://doi.org/10.1190/1.1441280

[18] Abate, J., Whitt, W., (1992). The Fourier-series method for inverting transforms of probability distributions. Queueing Systems. 10(1): 5-87. https://doi.org/10.1007/BF01158520

[19] Kou, S.G., Wang, H., (2003). First passage times of a jump diffusion process. Advances in Applied Probability: 504-31. https://doi. org/10.1239/aap/1051201658

[20] Badescu, A., Breuer, L., Da Silva Soares, A., Latouche, G., Remiche, M.-A., Stanford*, D., (2005). Risk processes analyzed as fluid queues. Scandinavian Actuarial Journal. 2005(2): 127-41. https://doi. org/10.1080/03461230410000565

[21] Schoutens, W., Van Damme, G., (2011). The $\beta$-variance gamma model. Review of Derivatives Research. 14(3): 263-82. https://doi. org/10.1007/s11147-010-9057-y

[22] Montella, C., (2008). LSV modelling of electrochemical systems through numerical inversion of Laplace transforms. I-The GS-LSV algorithm. Journal of Electroanalytical Chemistry. 614(1-2): 121-30. https://doi.org/10.1016/j.jelechem.2007.11.010

[23] Kawakatsu, H., (2005). Numerical inversion methods for computing approximate p-values. Computational Economics. 26(3-4): 10316. https://doi.org/10.1007/s10614-005-9011-5

[24] Egonmwan, A.O., (2012). The numerical inversion of the Laplace transform. LAP Lambert Academic Publishing

[25] Valkó, P.P., Vajda, S., (2002). Inversion of noise-free Laplace transforms: towards a standardized set of test problems. Inverse Problems in Engineering. 10(5): 467-83. https://doi. org/10.1080/10682760290004294

[26] Mashayekhizadeh, V., Dejam, M., Ghazanfari, M.H., (2011). The application of numerical Laplace inversion methods for type curve development in well testing: a comparative study. Petroleum Science and Technology. 29(7): 695-707. https://doi. org/10.1080/10916460903394060

[27] Zhang, J., (2007). Some innovative numerical approaches for pricing American options.

[28] Hassanzadeh, H., Pooladi-Darvish, M., (2007). Comparison of different numerical Laplace inversion methods for engineering applications. Applied Mathematics and Computation. 189(2): 1966-81. https://doi.org/10.1016/j.amc.2006.12.072

[29] Cheng, A.H.D., Sidauruk, P., Abousleiman, Y., (1994). Approximate inversion of the Laplace transform. Mathematica Journal. 4(2): $76-82$. 